\title{
Clinical trial of carbazepine (Tegretol) in trigeminal neuralgia
}

\author{
F. G. CAMPBELL, J. G. GRAHAM, AND K. J. ZILKHA \\ From The National Hospital, Queen Square, and the Neurological Departments Guy's Hospital, \\ London, and Cardiff Royal Infirmary
}

Carbazepine, Tegretol (5-carbamyl-dibenz (b,f)azepin), originally known as G.32883 and introduced in 1959 as an anticonvulsant, was first shown to have some effect in relieving the pain of trigeminal neuralgia by Blom $(1962,1963)$.

Since then Spillane (1963, 1964), McArdle (1963), and Taylor (1963) have confirmed that this drug has a specific effect in relieving the pain of trigeminal neuralgia in some 60 to $80 \%$ of patients.

This paper describes a controlled trial of carbazepine carried out simultaneously at The National Hospital, Queen Square, London, Cardiff Royal Infirmary, and Guy's Hospital, London.

\section{DESIGN OF TRIAL}

Patients were admitted to the trial without selection, except for a few who were rejected because of difficulty in attending regularly due to age, infirmity, or geography. Those whose pain was symptomatic of disseminated sclerosis were also excluded.

All patients included in the trial were in pain at the time of entry. Most had not previously received treatment with carbazepine, but the four who had had carbazepine before had been off the drug for at least two months before commencing the trial and were allocated, by chance, equally to the two treatment groups.

Patients were told that two drugs were to be compared for efficacy in relief of pain. The drugs were to be tried alternately for periods of two weeks at a time, and at the end of each fortnight the patient would be seen and questioned about his condition, any unused tablets would be returned, and the next fortnight's tablets issued.

The trial lasted eight weeks, i.e., the subjects passed two periods of alternate fortnights on each drug.

Carbazepine (C) and placebo (P) were issued by the pharmacists according to random number lists in the order C, P, C, P, or P, C, P, C, neither patient nor doctor knowing the order of therapy given.

The placebo tablets were made principally of lactose and were indistinguishable in appearance from the active drug. If a patient did not respond to treatment in one week and was in severe pain, he could return early and be changed over to the other treatment. There were four such patients; again two patients were in group $C, P, C, P$ and two in P, C, P, C.
Dosage at each visit began with half a tablet four times daily and if relief was inadequate after $\mathbf{4 8}$ hours, it could be increased to 1 tablet four times daily. This schedule was carried out at Guy's Hospital and the National Hospital, but at Cardiff, where a fatality associated with carbazepine had occurred, a maximum dosage of 3 tablets daily was observed, without apparent difference in the results.

At the initial visit information was recorded about age, sex, duration of the illness and number of previous bouts, previous treatment, the division of the trigeminal nerve involved, and the duration and severity of the current bout of pain.

At each subsequent visit enquiries were made about the severity of the pain (severe, moderate, mild), number of paroxysms daily and their duration, and the presence or absence of triggering of the pain by eating, talking, contact, draughts, and other factors. Finally enquiries were made for the known side effects of carbazepine and any others.

NUMBERS IN THE TRIAL AND COMPARISON OF THE TWO TREATMENT GROUPS

Seventy-seven patients were included in the trial at the outset. Of these seven were excluded subsequently for the following reasons: three failed to re-attend after the initial visit, the records of one were lost, one was an unreliable witness, one received the drugs in the wrong order, and one was withdrawn because of a rash after the first period.

Of the remaining 70 patients, 36 began on carbazepine and received their drugs in the order C, P, C, P, and 35 began on placebo and took the drugs in the order P, C, $\mathbf{P}, \mathbf{C}$.

The 70 patients ranged in age from 20 to 84 , the mean being 59 years, and $34 \%$ were males.

The two orders of treatment groups $(C, P, C, P$ and $P, C, P, C)$ were subsequently compared to ascertain whether or not they were similar in all important respects.

The groups were therefore compared for sex, age, number of previous bouts, duration of present bout, and division of nerve affected, and no striking difference was noted. As regards previous treatment, however, only $6 \%$ of the $\mathbf{C}, \mathbf{P}, \mathrm{C}, \mathbf{P}$ group had been injected for pain as against $29 \%$ of the P, C, P, C group. This obviously arose by chance and was not related to the initial distribution by categories of pain in the two groups (Table I). 
TABLE I

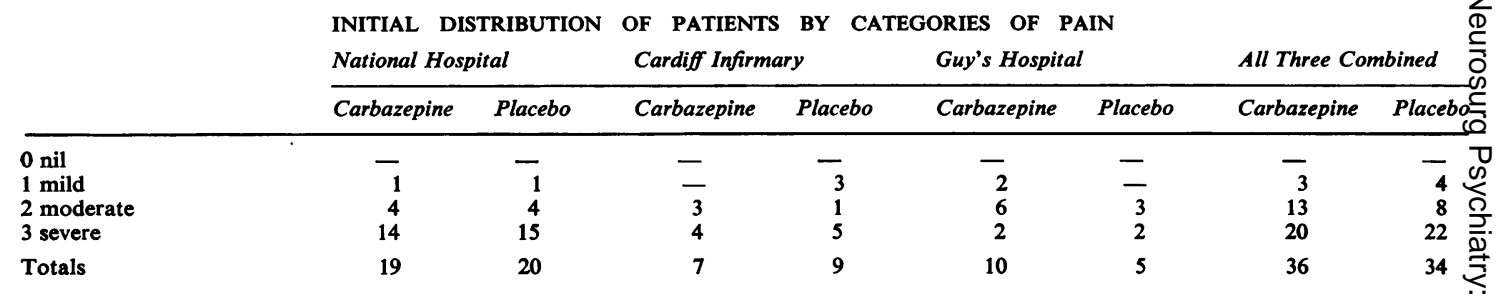

RESULTS

The results were assessed as regards severity of the pain, the number of paroxysms daily, and the effect of the drug on triggering mechanisms.

SEVERITY OF PAIN A system of scoring was devised for this purpose. Four categories of pain were recorded at each interview as follows: nil $=0$, mild $=1$, moderate $=2$, and severe $=3$.

If a patient began at 3 and moved at his next visit to 0 , he had scored +3 of a possible +3 , and his actual improvement expressed as a percentage of his possible improvement (or upgrading) was called $100 \%$. If, however, he moved from 2 to 1 , his score was +1 of a possible +2 , i.e., percentage upgrading $=50 \%$.

Similarly, if he deteriorated from 0 to 2 his score was -2 out of a possible -3 , and the actual downgrading expressed as a percentage of the possible downgrading $=66 \%$.

Thus, in each group the sum of the actual upgradings or downgradings was expressed as a percentage of the sum of the possible upgradings or downgradings.

These calculations showed that the group which began on carbazepine achieved $58 \%$ (51/89) upgrading in the first period, $5 \%(2 / 37)$ in the second period on placebo, $64 \%(38 / 59)$ in the third period on carbazepine, and $15 \%(4 / 26)$ in the last period on placebo. (The figures in brackets are the actual and possible total number of grades.)

The P,C,P,C group, however, was upgraded only $26 \%(22 / 86)$ in the first period on placebo, $41 \%$ $(27 / 66)$ in the second period on carbazepine, $17 \%$ $(7 / 41)$ in the third period on placebo, and $52 \%$ $(28 / 54)$ in the last period on carbazepine. Calculations for downgrading were made and showed a similar trend.

The figures were very greatly in favour of carbazepine. Both groups were upgraded more when on the drug than when on placebo, and were also downgraded more when on placebo than when on carbazepine.

It was also noted that the group who began on carbazepine improved $58 \%$ in their first period on the drug, while those who began on placebo im proved only $41 \%$ when on carbazepine for the firsto time, i.e., in their second fortnight. This difference ino response is unrelated to the initial degree of the severity of pain which, as has already been pointed. out, shows a roughly similar distribution in the twon groups (Table 1).

If one regards the first period of two weeks as $a \vec{\circ}$ traditional type of trial (and not 'cross-over') in which two groups with similar characteristics at the outset are given carbazepine or placebo in consecutive order, then the result of the trial is even more clear cut: the carbazepine group were upgraded $58 \%$ of aic possible 89 grades and downgraded $0 \%$ of a possibtew 19 , while the placebo group were upgraded by $26 \%$ of a possible 86 grades, and downgraded $12 \%$ onacr possible 16 . The difference of $32 \%$ in upgrading statistically significant $(P<0 \cdot 01)$. Because it is freefrom the ambiguities that may arise in the interpreta tion of cross-over procedures this is also a vafid conclusion to be derived from the data on the severty of pain in this trial.

NUMBER OF PAROXYSMS It was not found possible? to keep to exact numerical answers to questions about the number of paroxysms of pain, and the patients replies were therefore categorised $0,1,2$ or 3 , whereक $0=$ nil and $3=$ 'every half hour', 'innumerable', 'hundreds'. Using these categories as had been doneo for severity of pain, upgrading and downgrading were calculated and expressed as percentages of the amount possible. The carbazepine group improved $68 \%$ of the possible amount in the first period, and the placebo group only $26 \%$.

TRIGGER MECHANISMS The triggering effect on the pain by eating, talking, contact, and draughts was considered at each attendance, and when this information was collated at the end of the trial it was? possible to give the actual disappearance or appear $\rightarrow$ ance of triggering factors as percentages of the amount of disappearance or appearance possible. $N$ For all four triggering factors combined, the carbazepine group showed $68 \%$ upgrading as compared with $40 \%$ in the placebo group, and $2 \%$ downgrading 
as compared with $20 \%$, both statistically significant figures $(P=0.05)$.

A valid and simple comparison of the response of tic douloureux to carbazepine as against placebo seems to be in the first two weeks of the trial, comparing 36 patients on carbazepine with 34 on placebo. The results are summarized in Table II.

TABLE II

\section{SUMMARY OF RESULTS}

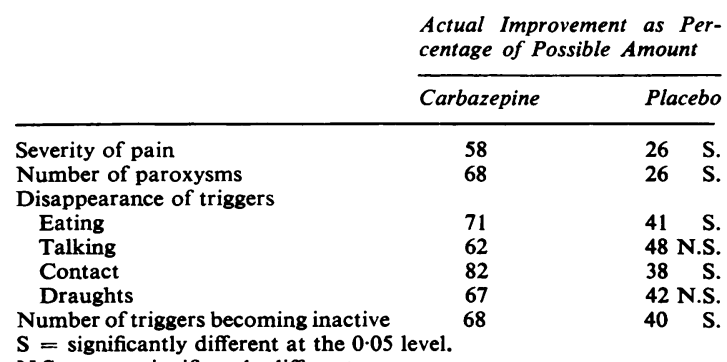

$\mathrm{S}=$ significantly different at the 0.05 level.

N.S. = not significantly different.

SIDE EFFECTS Fifty per cent of patients experienced at least one side effect on carbazepine as against $24 \%$ on placebo. In the case of carbazepine, giddiness was the most frequent side effect, occurring in $30 \%$ of patients in the first period on the drug and dropping to $23 \%$ during the second period. Unsteadiness and drowsiness were the next most frequent side effects, occurring in $15 \%$ of patients and not necessarily together.

The most important side effect, which was seen in this trial once only and led to the withdrawal from the trial of this patient, is the occurrence of a rash. In this case it was erythematous, but it can be morbilliform or urticarial. We regarded its appearance as an indication for stopping the drug. Two deaths from agranulocytosis have been reported in patients taking carbazepine and one had had a rash previously (Spillane, 1964; Donaldson and Graham, 1965).

\section{SUMMARY}

In a controlled trial, carbazepine has been shown to be much more effective than a placebo in the treatment of trigeminal neuralgia.

We wish to thank Dr. M. J. McArdle and Dr. J. D. Spillane for their help and encouragement in carrying out this trial; we are grateful to Dr. Lewis-Faning for his help in the analysis of the statistical data. Our thanks are due to Dr. Alan Galbraith, of the Medical Department, Geigy Pharmaceutical Company Limited, for generous supplies of carbazepine (Tegretol).

\section{REFERENCES}

Blom, S. (1962). Trigeminal Neuralgia: its treatment with a new anticonvulsant drug (G.32883). Lancet, 1, 839-840.

- (1963). Tic douloureux treated with new anticonvulsant. Experiences with G.32883. Arch Neurol. (Chic.), 9, 285-290.

Donaldson, G. W. K., and Graham, J. G. (1965). Aplastic anaemia following the administration of tegretol. Brit. J. clin. Pract., $19,699-702$.

McArdle, M. J. (1963). Personal communication.

Spillane, J. D. (1963). The treatment of trigeminal neuralgia with 'Tegretol'. In Proceedings of the Association British Neurologists, Harrogate, April.

- (1964). The treatment of trigeminal neuralgia. Preliminary experience with 'Tegretol'. Practitioner, 192, 71-77.

Taylor, J. C. (1963). Trigeminal neuralgia treated with G.32883. J. Neurol. Neurosurg. Psychiat., 26, 553-554. 